\title{
Functional and Evolutionary Implications of the Distribution of Phosphagens in Primitive-Type Spermatozoa
}

\author{
W. ROSS ELLINGTON ${ }^{1, *}$ AND STEPHEN T. KINSEY ${ }^{2}$ \\ ${ }^{1}$ Department of Biological Science and Institute of Molecular Biophysics, Florida State University, \\ Tallahassee, Florida; 32306-4370, and ${ }^{2}$ Department of Biological Science, University of North \\ Carolina, 601 South College Road, Wilmington, North Carolina 28403-3297
}

\begin{abstract}
External fertilization is considered to be the primitive condition in metazoans. The spermatozoa of such organisms typically display a common primitivetype morphology that is present in a range of phyla. These spermatozoa are extremely polarized cells in that the site of ATP synthesis (mitochondria in midpiece) is located at large diffusion distances from the ATP sink (dynein ATPases in the flagellum). Spermatozoa of polychaetes, sipunculids, echiuroids, echinoderms, and tunicates contain the phosphagen creatine phosphate or express the corresponding phosphagen kinase creatine kinase (or both), even when other phosphagens/phosphagen kinases are present in somatic tissues and eggs. The selective expression of the creatine kinase system in these spermatozoa may be related to potential advantages in the cellular transport of energy. To evaluate this possibility, we compared the efficacy of the major phosphagen systems for cellular transport of energy. We used a facilitated diffusion model for spatial ATP buffering, taking into account relative differences in diffusivity and thermodynamic poise. At low ratios of [total phosphagen pool]/ [total adenine nucleotide pool] $\left(\mathrm{C}_{\mathrm{G}+\mathrm{P}} / \mathrm{C}_{\mathrm{Ad}}\right.$ ratio), creatine phosphate carried a higher fraction of total high-energy phosphate (J) than the other phosphagens. However, J values for all phosphagens were greater than 0.9 , and these differences disappeared as the $\mathrm{C}_{\mathrm{G}+\mathrm{P}} / \mathrm{C}_{\mathrm{Ad}}$ ratio was increased. Thus, the functional benefit of using $\mathrm{CP}$, rather than other phosphagens, in energy transport is quite limited. The creatine kinase system became associated with primitive-type spermatozoa early in metazoan evolution.
\end{abstract}

Received 23 March 1998; accepted 9 September 1998.

* Author to whom correspondence should be addressed. E-mail: elling@bio.fsu.edu
This association is not necessarily related to inherent advantages of this phosphagen system for buffering of ATP, but may be linked to historical events in the evolution of the cell phenotype.

\section{Introduction}

The energy "content" of ATP is not fixed but rather is related to the extent of displacement of the hydrolysis reaction (ATP $\rightarrow$ ADP + inorganic phosphate) from thermodynamic equilibrium (Nicholls and Ferguson, 1992), as the effective free energy of ATP hydrolysis (ATP chemical potential, $\Delta \mathrm{G}_{\mathrm{ATP}}$ ) is a function of the physiological [ATP]/[ADP] ratio. Under normal conditions, cellular energy metabolism maintains the hydrolysis reaction far displaced from equilibrium, which is reflected by high prevailing [ATP]/[ADP] ratios and correspondingly high values for $\Delta G_{\text {ATP }}$ (Nicholls and Ferguson, 1992; Kammermeier, 1993). Physiological conditions that lead to increases in cellular ADP concentrations lead to disproportionately larger decreases in the $[\mathrm{ATP}] /[\mathrm{ADP}]$ ratio. The net effect is substantial reductions in $\Delta \mathrm{G}_{\text {ATP }}$ that dramatically impact the functioning of critical ATP-requiring processes in cells (Kammermeier et al., 1982; Kammermeier, 1987, 1993).

Phosphagens are phosphorylated guanidine compounds that are involved in buffering cellular ATP levels. These compounds are typically found in cells with high and variable rates of energy turnover. A diverse array of phosphagens is found in the animal kingdom, including arginine phosphate (AP), creatine phosphate (CP), glycocyamine phosphate (GP), taurocyamine phosphate (TP), hypotaurocyamine phosphate (HTP), and lombricine phosphate (LP). Corresponding phosphagen (guanidino) 
kinases - arginine kinase $[\mathrm{AK}]$, creatine kinase [CK], glycocyamine kinase [GK], taurocyamine kinase [TK], hypotaurocyamine kinase [HTK] and lombricine kinase [LK] - catalyze the reversible transfer of phosphate from phosphagen to ADP, yielding ATP (phosphagen + ADP $\leftrightarrow$ ATP + guanidine acceptor).

The traditional view of phosphagen systems is that they function as temporal ATP buffers (Meyer et al., 1984). Typically, phosphagen concentrations are higher than adenine nucleotide concentrations (Beis and Newsholme, 1975; Meyer et al., 1984). During periods of intense energy turnover, $\Delta \mathrm{G}_{\mathrm{ATP}}$ is kept high by the transphosphorylation of ADP to ATP at the expense of the phosphagen. This role of the phosphagen system has been likened to that of the discharge of a capacitor (Meyer, 1988; Kammermeier, 1993). Ellington (1989) showed that the apparent equilibrium constants $\left(\mathrm{K}^{\prime}=([\mathrm{ATP}] *\right.$ [guanidine] $) *$ $\left([\text { phosphagen] } *[\mathrm{ADP}])^{-1}\right.$ ) for the phosphagen kinase reactions span a range of values from $13.2(\mathrm{AK})$ to 100 (CK); others are intermediate. This means that the CK reaction can buffer ATP over higher ranges of $\Delta \mathrm{G}_{\mathrm{ATP}}$ than other phosphagen systems (Ellington, 1989). However, CP might be viewed as being disadvantageous in certain invertebrate systems in which lower $\mathrm{pH}$ values and reduced set-point $\Delta \mathrm{G}_{\mathrm{ATP}}$ values may prevail, thereby inducing rapid dissipation of the CP pool (Wyss et al., 1992).

A second hypothesized function of phosphagens is a potential role (particularly $\mathrm{CP}$ ) in the transport of highenergy phosphate from "source" (typically mitochondria) to "sink" (ATPases). One perspective on the transport role, formulated as the CP "shuttle" (Bessman and Geiger, 1981) and later CP "circuit" (Wallimann et al., 1992), hypothesizes that there are separate pools of adenine nucleotides at source and sink and that the CP/CK system provides the energetic link between the two sites. The net effect is to allow rapid transport of high-energy phosphate while maintaining appropriate $\Delta \mathrm{G}_{\mathrm{ATP}}$ valucs at source and sink (Mainwood and Rakuson, 1982; Jacobus, 1985; Kammermeicr, 1987). The above arguments are based on diffusive constraints (concentration gradients; membrane permeability) of the adenine nucleotides $v s$. $\mathrm{CP} /$ creatine in relation to energy turnover rates. The basic premise for this view is that intracellular diffusion of ATP and particularly ADP is limiting. That is, the conditions that optimize diffusive movement of the adenine nucleotides (high concentration gradients) would produce $\Delta G_{\text {ATP }}$ values at the site of ATP utilization (sink) that would be incompatible with functioning of the ATPase (Jacohus, 1985; Kammermeier, 1987). Furthermore, additional diffusive constraints for ADP, such as binding to intracellular surfaces and reduced permeability for entry into the mitochondrion, have been identified (Saks et al., 1993, 1996).

Phosphagens are distributed along distinct phylogenetic lines (for reviews see Watts, 1968, 1971, and 1975). The $\mathrm{CP} / \mathrm{CK}$ system is found in the vertebrates, lower chordates, and lower and higher invertebrate groups. The AP/AK system is widely distributed throughout the invertebrates and is present in lower chordates but absent in the vertebrates. The remaining phosphagen systems are primarily found in vermiform groups best typified by polychaetes, which as a group have all phosphagen systems (Watts, 1968, 1971, 1975). Of great interest is the observation that in such groups as polychaetes, echinoderms, and tunicates, regardless of which phosphagen system is present in somatic cells and eggs, the CP/CK system is always present in the primitive-type spermatozoa of these animals (Watts, 1968, 1971, 1975; Tombes and Shapiro, 1989). It has long been recognized that the diffusion of ATP (and ADP) might limit motility in cells that use flagella and cilia (Raff and Blum, 1968; Nevo and Rickenspoel, 1970; Lin, 1972). Thus, energy transport by phosphagens should be especially important in more highly polarized cells such as primitive-type spermatozoa, which have mitochondria in the midpiece and dynein ATPases distributed along the flagellum (Tombes and Shapiro, 1989). Here we evaluate differences in the energy transport properties of the various phosphagen systems in an effort to explain the selective expression of the CP/CK system in these primitive-type spermatozoa.

\section{Materials and Methods}

\section{Rationale for approach}

The functioning of the phosphagen systems in energy transport should be dependent on a number of parameters - thermodynamic properties of the phosphagen kinase reaction (Meyer et al., 1984; Ellington, 1989) and diffusive constraints such as relative diffusivities and concentration differences. Thermodynamic differences have been documented (Ellington, 1989). The various phosphagens span a range of relative molecular masses (Mr). According to the Stokes-Einstein equation, diffusivity (diffusion coefficient, D) is given by the following expression:

$$
\begin{aligned}
\mathrm{D}= & \mathrm{k} * \mathrm{~T} /(6 * \mathrm{P} * \mathrm{n} * \mathrm{r}) \\
& \text { where } \\
\mathrm{k}= & \text { Boltzman's constant, } \\
\mathrm{T}= & \text { absolute temperature, } \\
\mathrm{n}= & \text { viscosity } \\
\mathrm{r}= & \text { molecular radius }
\end{aligned}
$$

Molecular volume should be roughly proportional to $\mathrm{Mr}$. If one assumes a spherical molecule, then $D$ values for each phosphagen should be proportional to the cubed root of the Mr. In the present study we empirically determined 
the relative diffusivities of five major phosphagens and ATP. We then used these data to model the functional impact of differences in $\mathrm{D}, \mathrm{K}^{\prime}$, and phosphagen concentrations on energy transport, using the steady-state, facilitated diffusion model of Meyer et al. (1984), in which energy transport by phosphagens is viewed as spatial ATP buffering.

\section{Phosphagens}

Five major phosphagens were used in this study (see Fig. 1 for chemical structures and $\mathrm{Mr}$ valucs). $\mathrm{CP}$ and AP are available commercially from Sigma Chemical Co. (St. Louis, MO). The remaining phosphagens were enzymatically synthesized using the appropriate guanidine acceptor and phosphagen kinase. We followed the protocol of Poat et al. (1980) with an ATP-regenerating system consisting of phosphoenolpyruvate and pyruvate kinase. CK catalyzes the phosphorylation of glycocyamine, albeit at a much lower rate than creatine. Thus, GP was synthesized using glycocyamine (Sigma) and muscle CK (Boehringer Mannheim, Indianapolis, IN). LK catalyzes the phosphorylation of both lombricine and taurocyamine (Suzuki et al., 1997). LK was purified from the body wall of the earthworm Eisenia foetida according to the protocol of Suzuki et al. (1997). Lombricine was isolated as a natural product from the body wall of the earthworm Lumbricus terrestris according to the protocol of Ellington (1989). Purity was verified using thin-layer chromatography, NMR spectroscopy, and enzymatic assay. Taurocyamine was synthesized as previously described (Ellington, 1989). Both LP and TP were enzymatically synthesized using LK as described above. All other biochemical reagents were from Sigma or Boehringer Mannheim.

\section{Measurement of diffusion coefficients using pulsed gradient NMR spectroscopy}

Diffusion coefficients for phosphagens and ATP were determined using a Bruker DMX-WB600 NMR spectrometer equipped with XYZ gradients. Phosphagens and ATP were dissolved in a model solution consisting of 50 $\mathrm{m} M$ HEPES buffer, $200 \mathrm{~m} M \mathrm{KCl}, 100 \mathrm{~m} M$ glycine, and $25 \mathrm{~m} M \mathrm{MgCl}_{2}$ (pH of solution adjusted to 7.4). Samples were placed in 10-mm NMR tubes and mounted in a microimaging probe. Phosphorus NMR spectra were acquired at $242 \mathrm{MHz}$. Temperature was maintained at $20^{\circ} \mathrm{C}$. Diffusion coefficients were determined with pulsed gradient spin echo NMR (PGSE) using a bipolar gradient sequence with a longitudinal eddy current delay period (BPP-LED) (Wu et al., 1995). In this protocol, spin echo experiments were conducted with increasing applied gradients that resulted in attenuation of the ${ }^{31} \mathrm{P}-\mathrm{NMR}$ signal. The slope of this attenuation curve is directly proportional to the mobility of the molecule being observed. Due to this dependence on mobility, it was possible to calculate the D values under these circumstances. Since AP, GP, TP, and LP have nearly identical chemical shifts, these phosphagens were investigated in independent experiments.

\section{Mathematical treatments}

Numerical simulations were conducted using Mathcad Plus version 6 (Mathsoft, Cambridge, MA) on a Pow-
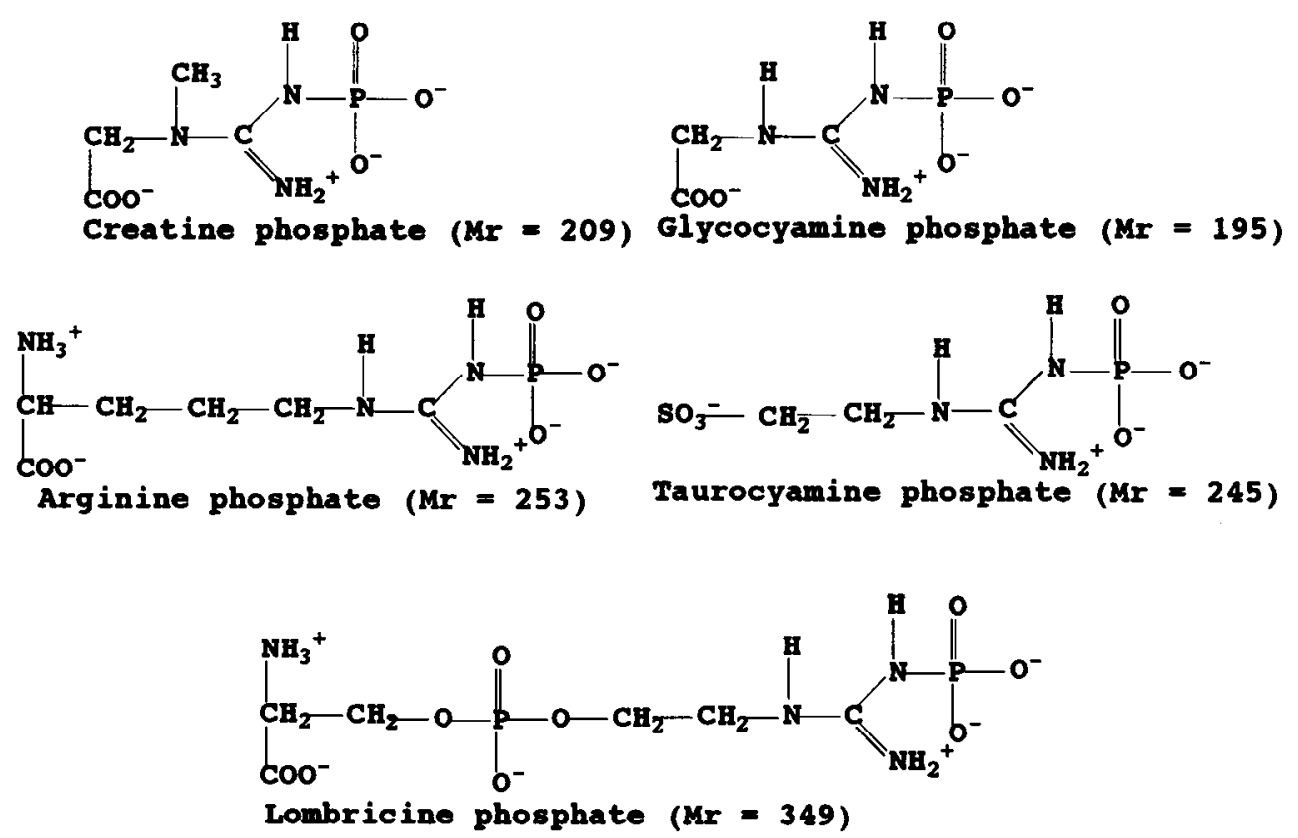

Figure 1. Structures of five of the major phosphagens. 
erMac 7600 microcomputer and SlideWrite Plus version 4 (Advanced Graphics Software, Carlsbad, CA) on a Pentium $133 \mathrm{MHz}$ microcomputer. Equations and boundary conditions are described in the text.

\section{Results and Discussion}

\section{Diffusion coefficient}

PGSE NMR determinations of diffusion coefficients for phosphagens and ATP yielded very consistent results within replicates for each treatment (Table I; Fig. 2). As cxpected according to the Stokes-Einstein equation, the $\mathrm{D}$ values were indirectly proportional to $\mathrm{Mr}$. That is, there was a linear relationship between the diffusion coefficient and the reciprocal of the cube root of the $\mathrm{Mr}$ (Fig. 2). In the context of cellular energy transport, the more physiologically relevant parameter is the ratio $\mathrm{D}_{\text {phosphagen }} / \mathrm{D}_{\mathrm{ATP}}$, which reflects relative mobilities of the "high energy" phosphates. The ratios range from 1.222 for the largest phosphagen, LP, to 1.597 for the smallest, GP (Table I). The $\mathrm{D}_{\mathrm{PC}} / \mathrm{D}_{\mathrm{ATP}}$ ratio observed in the present study is highly comparable to values obtained in model solutions by other groups (Yoshizaki et al., 1987; Moonen et al., 1990; Hubley et al., 1995).

\section{Simulation of spatial ATP buffering}

Meyer et al. (1984) pointed out that under conditions of submaximal rates of aerobic energy turnover, the role of phosphagens can be viewed as a form of facilitated diffusion in which the phosphagen and its corresponding guanidine base, in effect, substitute for ATP and ADP, respectively. If one assumes that the phosphagen kinase reaction is globally near equilibrium throughout the cell,

Tahle I

Properties of phosphagens and corresponding phosphagen kinase reactions; data for diffusion coefficients $(D)$ correspond to means $\pm 1 S D$

\begin{tabular}{|c|c|c|c|}
\hline & $\mathrm{D}\left(\mathrm{cm}^{2} / \mathrm{s}\right) \times 10^{-6}$ & $\begin{array}{l}\text { Dphosphagen/ } \\
\text { DATP }\end{array}$ & $\begin{array}{l}\text { Apparent equilibrium } \\
\text { constant }^{1}\end{array}$ \\
\hline ATP & $\begin{array}{c}2.872 \pm 0.212 \\
(n=23)\end{array}$ & - & - \\
\hline $\begin{array}{l}\text { Lombricine } \\
\text { phosphate }\end{array}$ & $\begin{array}{c}3.509 \pm 0.184 \\
(n=12)\end{array}$ & 1.222 & 32.3 \\
\hline $\begin{array}{l}\text { Arginine } \\
\text { phosphate }\end{array}$ & $\begin{array}{c}4.054 \pm 0.129 \\
(n=5)\end{array}$ & 1.412 & 13.2 \\
\hline $\begin{array}{l}\text { Taurocyamine } \\
\text { phosphate }\end{array}$ & $\begin{array}{c}4.169 \pm 0.063 \\
(n=6)\end{array}$ & 1.452 & 27.3 \\
\hline $\begin{array}{l}\text { Creatine } \\
\text { phosphate }\end{array}$ & $\begin{array}{c}4.329 \pm 0.089 \\
(n=5)\end{array}$ & 1.508 & 100 \\
\hline $\begin{array}{l}\text { Glycocyamine } \\
\text { phosphate }\end{array}$ & $\begin{array}{c}4.586 \pm 0.177 \\
(n=6)\end{array}$ & 1.597 & 29.0 \\
\hline
\end{tabular}

\footnotetext{
${ }^{1}$ From Ellington (1989).
}

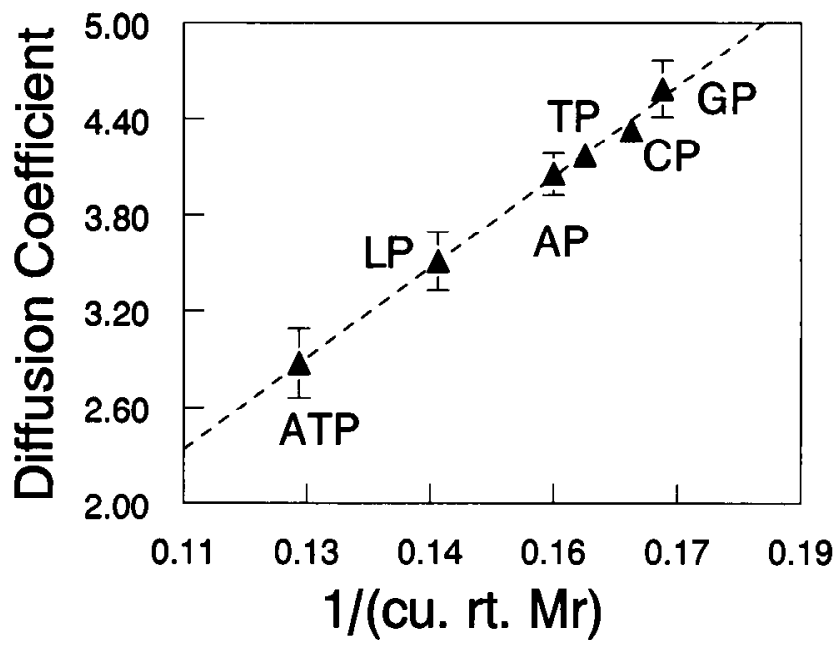

Figure 2. Relationship between diffusion coefficient $\left(\times 10^{-6} \mathrm{~cm}^{2} / \mathrm{s}\right)$ and the cubed root of the relative molecular mass. Each value represents a mean $+1 \mathrm{SD}$ ( $n$ size is reported in Table I).

then the importance of phosphagens in energy transport can be described by the following relation (Meyer et al., 1984):

$$
\begin{array}{r}
\mathbf{J}_{\text {phos. }} / \mathbf{J}_{\mathrm{ATP}}=\left(\mathrm{D}_{\text {phosphagen }} / \mathrm{D}_{\mathrm{ATP}}\right) *\left(\mathrm{C}_{\mathrm{G}+\mathrm{P}} / \mathrm{C}_{\mathrm{Ad}}\right) \\
* \mathrm{~K}^{\prime} *\left[(\mathrm{R}+1) /\left(\mathrm{R}+\mathrm{K}^{\prime}\right)\right]^{2}
\end{array}
$$

where

$\mathrm{J}_{\text {phos. }} / \mathrm{J}_{\mathrm{ATP}}=$ ratio of phosphate carried by the phosphagen over phosphate carried by ATP

$\mathrm{C}_{\mathrm{G}+\mathrm{P}} / \mathrm{C}_{\mathrm{Ad}}=$ ratio of concentration of total phosphagen pool over total adenine nucleotide pool

$\mathrm{K}^{\prime}$ = apparent equilibrium constant of phosphagen kinase

$\mathrm{R}=[\mathrm{ATP}] /[\mathrm{ADP}]$ ratio

In this context, it is assumed that the diffusion coefficients for phosphagen $v s$. guanidine base and ATP vs. ADP are not substantially different (Meyer et al., 1984). Implicit in our use of in vitro $\mathrm{D}$ values and $\mathrm{D}_{\text {phosphagen }} / \mathrm{D}_{\mathrm{ATP}}$ ratios is the assumption that these ratios do not change appreciably in vivo. This assumption appears to be valid for $\mathrm{D}_{\mathrm{PC}} /$ $\mathrm{D}_{\text {ATP }}$ in vertebrate skeletal muscle (Hubley et al., 1995). The Meyer et al. (1984) model was originally developed to look at high-energy phosphate diffusion in skeletal muscle fibers. Although the geometry of spermatozoa is somewhat different, we consider the application of the model appropriate in the context of comparing relative capacities for energy transport amongst the phosphagens.

For the purposes of establishing boundary conditions, we chose a physiologically reasonable value of 300 for the $[\mathrm{ATP}] /[\mathrm{ADP}]$ ratio. Boundary limits for $\mathrm{D}_{\text {phosphagen }} /$ $D_{\text {ATP }}$ ratios and $K^{\prime}$ were 1.1 to 1.6 and 10 to 100 , respectively, which bracket the range of values in Table I. For 
values of the $\mathrm{C}_{\mathrm{G}+\mathrm{P}} / \mathrm{C}_{\mathrm{Ad}}$ ratio we chose a range of values of 1 to 8 , which brackets the observed pool ratios for a range of contractile/motile cells (Beis and Newsholme, 1975; Tombes et al., 1985; Hamm and Yue, 1987; Schneider et al., 1989). Numerical solutions to the above equation were calculated and expressed as the fraction of total high-energy phosphate flux carried by the phosphagen $(\mathrm{J})$.

Figure 3 depicts the relationship between $\mathrm{J}, \mathrm{D}_{\text {phosphagen }} /$ $\mathrm{D}_{\mathrm{ATP}}$ ratio, and $\mathrm{K}^{\prime}$ at a $\mathrm{C}_{\mathrm{G}+\mathrm{P}} / \mathrm{C}_{\mathrm{Ad}}=1$ and a $[\mathrm{ATP}] /[\mathrm{ADP}]$ ratio $=300$. It is apparent that there is a steep dependence of $\mathrm{J}$ on $\mathrm{K}^{\prime}$, especially in the lower $\mathrm{K}^{\prime}$ range. The dependence of $\mathrm{J}$ on relative diffusivity is much less pronounced (Fig. 3). When one plots the individual 3-dimensional coordinates for each phosphagen type on the response surface, the positions of each are well dispersed (Fig. 3). However, CP's position on the surface shows that it carries the highest fraction of phosphate while AP carries the lowest. The other three phosphagens show intcrmediate $\mathbf{J}$ values (Fig. 3). Note that under the conditions used in the Figure 3 simulation, all of the phosphagens are responsible for transporting over $90 \%$ of high-energy phosphate. The impact of incrementally increasing $\mathrm{C}_{\mathrm{O}+\mathrm{P}} / \mathrm{C}_{\mathrm{Ad}}$ ratios on $\mathbf{J}$ is shown in Figure 4. The dependence of $\mathbf{J}$ on the $\mathrm{D}_{\text {phosphagen }} / \mathrm{D}_{\mathrm{ATP}}$ ratio and $\mathrm{K}^{\prime}$ becomes increasingly compressed so that these latter two parameters have minimal impact at higher $\mathrm{C}_{\mathrm{G}+\mathrm{P}} / \mathrm{C}_{\mathrm{Ad}}$ ratios.

The above analyses were conducted using the limiting condition of the ATP/ADP ratio being equal to 300 . Although within physiological limits, this ratio reflects an upper extreme of energy state condition that may exist in

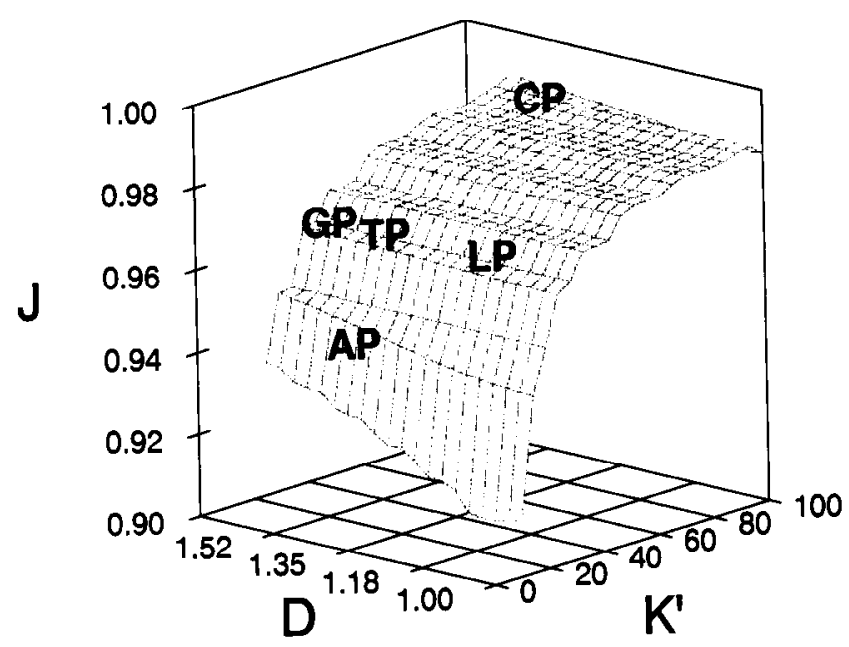

Figure 3. Impact of $D_{\text {phosphagen }} / D_{A T P}$ ratio (denoted $D$ in figure) and apparent equilibrium constant $\left(\mathrm{K}^{\prime}\right)$ on the fraction of total high-energy phosphate $(\mathrm{J})$ carried by phosphagens according to the facilitated diffusion model of Meyer et al. (1984). ATP/ADP and $\mathrm{C}_{\mathrm{G}+\mathrm{P}} / \mathrm{C}_{\mathrm{Ad}}$ ratios were set at 300 and 1 , respectively. Specific $J$ values for the various phosphagens (CP, GP, TP, LP, and AP) were calculated based on their respective $\mathrm{D}$ and $\mathrm{K}^{\prime}$ values. The positions of these phosphagens are indicated on the response surface. cells. Thus, we modeled $\mathrm{J}$ at a range of ATP/ADP ratios $(10-300)$. The results (Table II) show that differences in the transport capabilities of the phosphagens are minimal except at the lowest ATP/ADP ratio (10). In this extreme case, the CK system is the least effective in transport, as was first noted by Meyer et al. (1984).

Before assessing the physiological implications of these results, it is useful to comment on the validity of the above simulation. In addition to the facilitated diffusion approach, high-energy phosphate transport in vivo has also been investigated extensively using reaction-diffusion models (Raff and Blum, 1968; Nevo and Rikenspoel, 1970; Lin, 1972; Tombes et al., 1985; Hubley et al., 1997); these models require the use of in vivo D values that are typically somewhat lower than corresponding in vitro D values (Moonen et al., 1990; Hubley et al., 1995). Furthermore, intracellular barriers dramatically decrease in vivo $\mathrm{D}$ values in a time-dependent manner (Kinsey et al., 1998), which must be taken into account in reactiondiffusion analyses of energy transport. However, in our analyses we used $D_{\text {phosphagen }} / \mathrm{D}_{\mathrm{ATP}}$ ratios that should be minimally affected by such restrictions to diffusion and, consequently, would not compromise our overall conclusions.

Furthermore, the facilitated diffusion model is applicable only to situations in which aerobic energy turnover is near or below maximal and the global cellular highenergy phosphate concentrations do not change appreciably (or at least reach a new steady state). These circumstances should prevail in primitive-type spermatozoa of externally fertilizing marine animals that must swim for a considerable time in the water column. Recently, van Dorsten et al. (1997) showed that activation of motility in spermatozoa from the sea urchin Psammechinus miliaris is accompanied by dramatic increases in both respiration and the forward flux of $\mathrm{CK}(\mathrm{CP} \rightarrow \mathrm{ATP})$. In the same study, CP and ATP remained essentially constant for at least $2 \mathrm{~h}$ of motility, an observation consistent with steady-state energy transport by the $\mathrm{CP} / \mathrm{CK}$ system according to the facilitated diffusion model.

Phosphagen kinase equilibrium state and compartmentation arc other issues to be considered. The Meyer et al. (1984) approach assumes global equilibrium of the phosphagen kinase reaction throughout the cell. A number of workers have questioned the validity of this assumption (Saks and Aliev, 1996; Aliev and Saks, 1997). Recent evidence suggests that a significant fraction of the pool of creatine/creatine phosphate is not in equilibrium with CK in skeletal muscle fibers (Hochachka and Mossey, 1998). Furthermore, the whole issue of diffusion as a paradigm for intracellular transport has recently been questioned (Agutter et al., 1995). In some sense, these issues are experimentally intractable in the sperm system and cannot be addressed in this study. However, on the basis of cell morphology and rates of energy turnover, it 

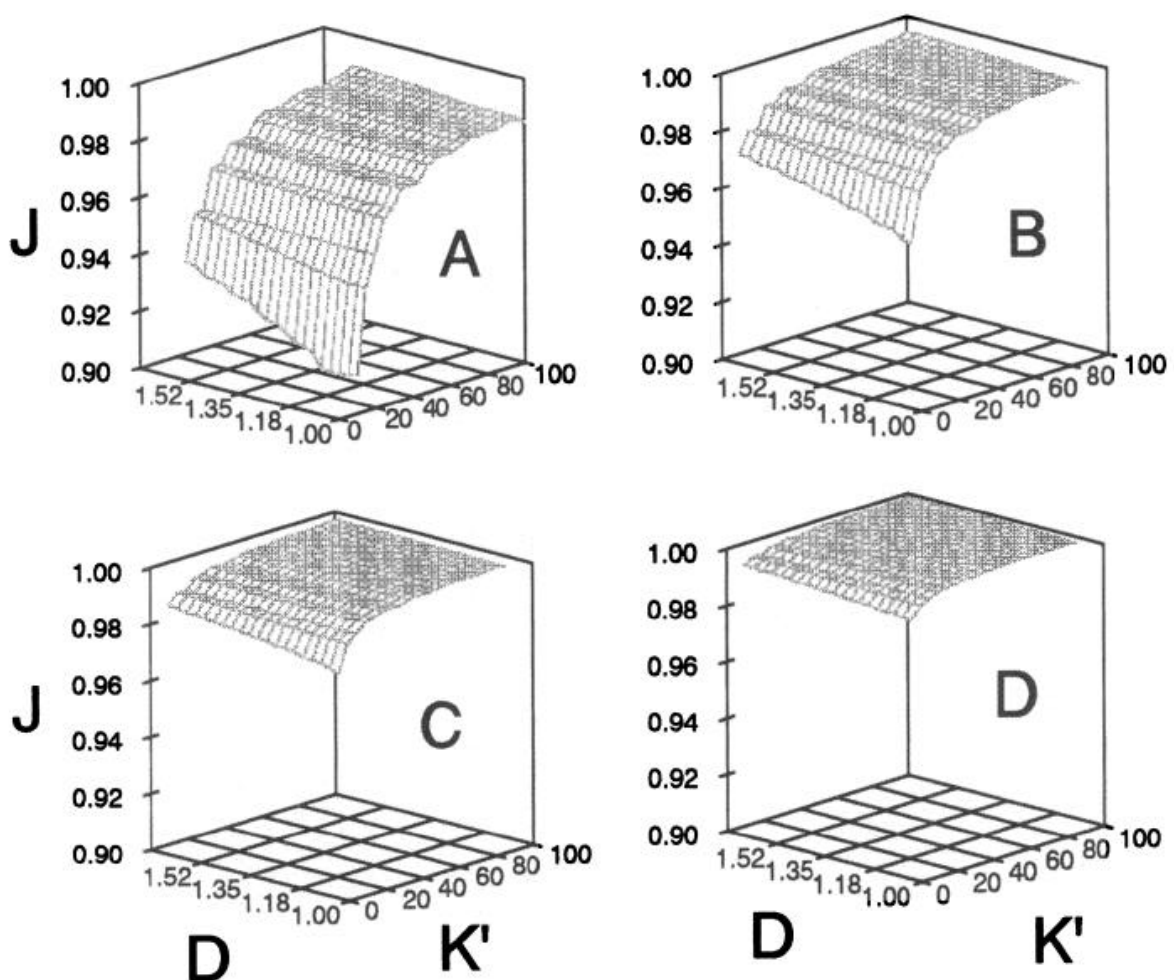

Figure 4. Impact of changes in the $\mathrm{C}_{\mathrm{G}+\mathrm{P}} / \mathrm{C}_{\mathrm{Ad}}$ ratio on fraction high-energy phosphate transport by phosphagens: (A) $\mathrm{C}_{\mathrm{G}+\mathrm{P}} / \mathrm{C}_{\mathrm{Ad}}=1$; (B) $\mathrm{C}_{\mathrm{G}+\mathrm{P}} / \mathrm{C}_{\mathrm{Ad}}=2$; (C) $\mathrm{C}_{\mathrm{G}+\mathrm{P}} / \mathrm{C}_{\mathrm{Ad}}=4$; and (D) $\mathrm{C}_{\mathrm{G}+\mathrm{P}} / \mathrm{C}_{\mathrm{Ad}}=8$. ATP/ADP ratio and $\mathrm{Y}$ and $\mathrm{X}$ axes are the same as in Fig. 3.

seems clear that there are certain diffusion restrictions on energy transport in primitive-type spermatozoa. Thus, in the present study we use the facilitated diffusion model to look only for broadscale and relative differences between the phosphagen systems-differences that would change the overall diffusive component of energy transport.

\section{Diffusion of phosphagens in physiological context}

The above results show that differences in thermodynamic potential and intrinsic diffusivity impact the rela-

Table II

Impact of changes in the setpoint ATP/ADP ratio on the transport capacities of phosphagen kinase reactions

\begin{tabular}{cccccc}
\hline \hline ATP/ADP ratio & LK & AK & TK & CK & GK \\
\hline 10 & 0.870 & 0.913 & 0.896 & 0.791 & 0.902 \\
25 & 0.953 & 0.956 & 0.961 & 0.942 & 0.964 \\
50 & 0.974 & 0.968 & 0.977 & 0.977 & 0.979 \\
100 & 0.982 & 0.974 & 0.984 & 0.990 & 0.986 \\
200 & 0.987 & 0.976 & 0.987 & 0.994 & 0.989 \\
300 & 0.988 & 0.977 & 0.989 & 0.995 & 0.990 \\
\hline
\end{tabular}

For the purpose of these calculations, the [total phosphagen pool]/ [total adenine nucleotide pool] $\left(\mathrm{C}_{\mathrm{G}+\mathrm{P}} / \mathrm{C}_{\mathrm{Ad}}\right.$ ratio $)$ was set at 2.5. Data are listed as fraction of the total high-energy phosphate carried by the phosphagen (J, as in Figures 3 and 4). tive capacity of phosphagens in facilitated energy transport under typical conditions of $\Delta \mathrm{G}_{\mathrm{ATP}}$. At lower $\mathrm{C}_{\mathrm{G}+\mathrm{P}} /$ $\mathrm{C}_{\mathrm{Ad}}$ ratios, $\mathrm{CP}$, because of its higher diffusivity and high $\mathrm{K}^{\prime}$, carries a greater fraction of high-energy phosphate $(\mathrm{J})$ than other phosphagens. However, even at low $\mathrm{C}_{\mathrm{G}+\mathrm{P}} / \mathrm{C}_{\mathrm{Ad}}$ ratios $\mathrm{J}$ values for all phosphagens fall well above 0.9 . Furthermore, these differences become negligible as the $\mathrm{C}_{\mathrm{G}+\mathrm{P}} / \mathrm{C}_{\mathrm{Ad}}$ ratio is increased (Fig. 4). Meyer et al. (1984) pointed out that the spatial ATP buffering role of phosphagens should be "most important in large cells with nonuniform mitochondrial distribution in which diffusion distances are greatest." Our analyses above show that spatial ATP buffering is moderately dependent on phosphagen type but strongly influenced by the $\mathrm{C}_{\mathrm{G}+\mathrm{P}} / \mathrm{C}_{\mathrm{Ad}}$ ratio. As indicated previously, primitive-type spermatozoa are highly polarized, with mitochondria separated by large diffusion distances from the dynein ATPases in the flagellum; yet these cells are capable of sustained aerobic energy turnover during swimming. Elegant work by Tombes and Shapiro (1985) and Tombes et al. (1985) showed that sea urchin spermatozoa contain creatine kinase (CK) and that sustained swimming is highly dependent on CK activity, presumably due to its role in energy transport.

The CP/CK system is known to be associated with primitive-type spermatozoa. Even though other phosphagen systems may be present in somatic tissues and eggs, 
the spermatozoa of all echinoderms, tunicates, and polychaetes consistently have the CP/CK system only (Watts, 1971; Tombs and Shapiro, 1989; Kamp et al., 1995), with $\mathrm{CK}$ activitics ranging from 7.1 to $30 \mu$ moles $* \mathrm{~min}^{-1} *$ $\mathrm{mg}$ protein $^{-1}$ in echinoderms (Ratto et al., 1989). The sipunculid Sipunculus nudus has high activities of AK in its somatic tissues (van Thoai et al., 1966) yet the spermatozoa contain CK only (G. Kamp, pers. comm.). Recently, we have shown that sperm of another sipunculid, Phascolopsis gouldii, contain significant CK activity (16.6 $\mu$ moles $* \min ^{-1} *$ mg protein ${ }^{-1}$ ) while muscle contains HTK activity (W. R. Ellington, unpub. obs.). This phenomenon is also characteristic of sperm of the echiuroid Urechis caupo, in which CK activity approaches 10 $\mu$ moles $* \min ^{-1} *$ mg protein ${ }^{-1}$ (W. R. Ellington, unpub. obs.) even though the phosphagen system present in muscle is LP/LK (Robin, 1964; van Thoai et al., 1972).

The above pattern, as seen in major protostome (polychaetes, echiuroids, and sipunculids) and deuterostome (echinoderms, tunicates) groups, indicates that when an organism has the potential to express more than one phosphagen type, if CP/CK is one of the possibilities, it is the type that will, without exception, be expressed in spermatozoa. Thus, the selective expression of the $\mathrm{CP} /$ CK system in cells where spatial ATP buffering is critical (using logic of Meyer et al., 1984) could be related to adaptive differences in functional propertics amongst the phosphagen systems.

The above adaptationist interpretation of $\mathrm{CP} / \mathrm{CK}$ system distribution in primitive-type spermatozoa is completely undermined by our analyses, above, which show that the differences in transport properties of the various phosphagen systems are small and that these differences collapse as the $\mathrm{C}_{\mathrm{G}+\mathrm{P}} / \mathrm{C}_{\mathrm{Ad}}$ ratio is increased. Marine bivalve molluscs have highly motile primitive-type spermatozoa that contain high AK activities (Tombes and Shapiro, 1989). The motility of these spermatozoa does not appear to be compromised by the lack of the CK/CP system. In 1971, Watts pointed out a distinct advantage for sperm to contain CP instead of AP: creatine is a dead-end compound, whereas arginine is in the mainstream of amino acid metabolism and is clearly important in sperm due to histone biosynthesis. This argument is credible for the preference of $\mathrm{CP}$ over AP in many spermatozoa but fails to explain the exclusion of phosphagens derived from glycocyamine, taurocyamine, hypotaurocyamine, and lombricine, all of which are dead-end compounds.

\section{Why is $C K$ selectively expressed in many spermatozoa?}

Spermatozoa demonstrate a diverse array of morphologies and metabolic capacities that correlate well with mode of fertilization and-for species with external fertilization - the nature of the microhabitat (Kamp et al., 1996; Kaldis et al., 1997). For instance, mammalian sper- matozoa have very low or no CK activity but generally have a high capacity for fermentative pathways (Kamp et al., 1996; Kaldis et al., 1997), which decreases the effective polarity ATP source and sink. Carp sperm have low CK activity but show motility for only a few minutes after release (Kamp et al., 1996). In contrast, phosphagen kinase activities (especially CK) are much higher in the sperm of externally fertilizing marine invertebrates (Tombes and Shapiro, 1989; Kamp et al., 1996). This dependency on phosphagen kinase is best exemplified by echinoderm sperm, which rely exclusively on mitochondrial fat oxidation (Kaldis et al., 1997) and must sustain long periods of motility in the water column.

Regardless of which model is utilized, the constraints on the diffusion of adenine nucleotides make phosphagen systems critical in the motility of many primitive-type spermatozoa. However, the present simulations suggest that the various phosphagen systems differ only slightly in their capacities for energy transport, as modeled in the context of facilitated diffusion, and that these differences collapse when the $\mathrm{C}_{\mathrm{G}+\mathrm{P}} / \mathrm{C}_{\mathrm{Ad}}$ ratio is increased (see Fig. 4). Thus, we are hard-pressed to ascribe a functional advantage in terms of energy transport to the possession of the $\mathrm{CP} / \mathrm{CK}$ system rather than another phosphagen system.

Echinoderm spermatozoa contain an octameric mitochondrial CK isoform (Tombes and Shapiro, 1985; Wyss et al., 1995) that is very similar to the vertebrate mitochondrial CK present in somatic tissues (Wyss et al., 1992, 1995). A unique "contiguous" trimeric CK is found restricted to the flagellum (Tombes and Shapiro, 1985; Wothe et al., 1990). We recently discovered that the spermatozoa of the polychaete Chaetopterus variopedatus have octameric mitochondrial and "contiguous" trimeric flagellar CKs which have strikingly similar physical properties to echinoderm sperm CKs (Ellington et al., 1998). Although definitive conclusions about the evolutionary relationships amongst these mitochondrial and flagellar CK's must await completion of our determinations of cDNA sequences for the polychaete forms, it seems likely that both the mitochondrial and flagellar CK isoforms evolved prior to the divergence of protostomes and deuterostomes, at least 670 million years ago (Doolittle et al., 1996).

A number of workers have recently pointed out the uncertainties of attempting to view physiological characters in cxtant animals as adaptations to current environmental situations (Huey, 1987; Harvey and Pagel, 1991). Historical issues have to be considered. External fertilization is thought to be the primitive metazoan condition (Baccetti and Afzelius, 1976) and primitive-type spermatozoa are very similar in structure regardless of group. It is tempting to speculate that the expression of the CK system may reflect some ancient linkage between the cell 
phenotype and the expression of the suite of cell-specific genes, including the two $\mathrm{CK}$ isoforms.

\section{Acknowledgments}

This research was supported by a grant from the National Science Foundation (IBN 96-31907) to WRE.

\section{Literature Cited}

Agutter, P. S., P. C. Malone, and D. N. Wheatley. 1995. Intracellular transport mechanisms: a critique of diffusion theory. $J$. Theor. Biol. 176: $26 \mathrm{I}-272$.

Aliev, M. K., and V. A. Saks. 1997. Compartmentalized energy transfer in cardiomyocytes: use of mathematical modeling for analysis of in vivo regulation of respiration. Biophys. J. 73: 428-445.

Bacetti, B., and B. A. Afzelius. 1976. The Biology of the Sperm Cell. Karger, Basel.

Beis, I., and E. A. Newsholme. 1975. The contents of adenine nucleotides, phosphagens and some glycolytic intermediates in resting muscles from vertebrates and invertebrates. Biochem. J. 102: 23-32.

Bessman, S. P., and P. J. Geiger. 1981. Transport of energy in muscle: the phosphorylcreatine shuttle. Science 211: 448-452.

Doolittle, R. F., D-F Feng, S. Tsang, G. Cho, and E. Little. 1996. Determining the divergence times of the major animal kingdoms of living organisms with a protein clock. Science 271: 470-477.

Ellington, W. R. 1989. Phosphocreatine represents a thermodynamic and functional improvement over other muscle phosphagens. J. Exp. Biol. 143: $177-194$

Ellington, W. R., K. Roux, and A. Pineda. 1998. Origin of octameric creatine kinases. FEBS Lett. 425: 75-78.

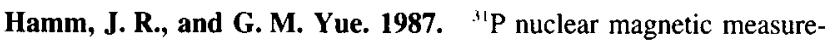
ments of intracellular $\mathrm{pH}$ in giant barnacle muscle. Am. J. Physiol. 252: C30-C37.

Harvey, P. H., and M. D. Pagel. 1991. The Comparative Method in Evolutionary Biology. Oxford University Press, Oxford.

Hochachka, P. W., and M. K. Mossey. 1998. Does muscle creatine phosphokinase have access to the total pool of phosphocreatine plus creatine? Am. J. Physiol. 274: R868-R872.

Hubley, M. J., R. C. Rosanske, and T. S. Moerland. 1995. Diffusion coefficients of ATP and creatine phosphate in isolated muscle: pulsed gradient ${ }^{3 /}$ P NMR of small biological samples. NMR Biomed. 8: $72-$ 78.

Hubley, M. J., B. R. Locke, and T. S. Moerland. 1997. Reactiondiffusion analysis of the effects of temperature on high-energy phosphate dynamics in goldfish skeletal muscle. J. Exp. Biol. 200: 975988

Huey, R. B. 1987. Phylogeny, history and the comparative method. Pp. 76-97 in New Directions in Ecological Physiology, M. E. Feder, A. F. Bennett, W. E. Burggren, W. W. and R. B. Huey, eds. Cambridge University Press, Cambridge.

Jacobus, W. E. 1985. Theoretical support for the heart phosphocreatine energy transport shuttle based on the intracellular diffusion limited mobility of ADP. Biochem. Biophys. Res. Commun. 133: $1035-1041$

Kaldis, P., G. Kamp, T. Piendl, and T. Wallimann. 1997. Functions of creatine kinase isoenzymes in spermatozoa. Adv. Devel. Biol. 5: $275-312$.

Kammermeier, H. 1987. Why do cells need phosphocreatine and a phosphocreatine shuttle? J. Mol. Cell. Cardiol. 19: 115-118.

Kammermeier, H. 1993. Meaning of energetic parameters. Basic Res. Cardiol. 88: $380-384$.

Kammermeier, H., P. Schmidt, and E. Jungling. 1982. Free energy change of ATP-hydrolysis: a causal factor of early hypoxic failure of the myocardium? J. Mol. Cell. Cardiol. 14: 267-277.
Kamp, G., H. Englisch, R. Muller, and D. Westhoff. 1995. Comparison of the two different phosphagen systems in the lugworm Arenicola marina. J. Comp. Physiol. B 165: 496-505.

Kamp, G., G. Busselmann, and J. Lauterwein. 1996. Spermatozoa: models for studying aspects of energy metabolism. Experientia 52: 487-494.

Kinsey, S. T., B. R. Locke, B. Penke, and T. S. Moerland. 1998. Diffusional anisotropy is induced by subcellular barriers in skeletal muscle. NMR Biomed. (in press).

Lin, S. H. 1972. Transient analysis of dual enzyme reaction in cilia Biophysik 8: $264-270$.

Mainwood, G. W., and K. Rakusan. 1982. A model for intracellular energy transport. Can. J. Physiol. Pharmacol. 66: 98-102.

Meyer, R. A. 1988. A linear model of muscle respiration explains monoexponential phosphocreatine changes. Am. J. Physiol. 254: C548-C553.

Meyer, R. A., H. L. Sweeney, and M. J. Kushmerick. 1984. A simple analysis of the 'phosphocreatine shuttle.' Am. J. Physiol. 246: C365-C377.

Moonen, C. T., P. C. M. van Zihl, D. Le Bihan, and D. DesPres. 1990. In vivo NMR diffusion spectroscopy: ${ }^{31} \mathrm{P}$ application to phosphorus metabolites in muscles. Magn. Reson. Med. 13: 467-477.

Nevo, A. C., and R. Rickenspoel. 1970. Diffusion of ATP in sperm flagella. J. Theoret. Biol. 26: 11-18.

Nicholls, D. G., and S. J. Ferguson. 1992. Bioenergetics 2, Academic Press, San Diego, California.

Poat, P. C., I. G. Giles, and K. A. Munday. 1980. An investigation into the apparent inhibition by arginine phosphate of the activity of Carcinus maenas type-M pyruvate kinase. Biochim. Biophys. Acta 613: $410-419$.

Raff, E. C., and J. J. Blum. 1968. A possible role for adenylate kinase in cilia: concentration profiles in a geometrically constrained dual enzyme system. J. Theor. Biol. 18: 53-71.

Ratto, A., B. M. Shapiro, and R. Christen. 1989. Phosphagen kinase evolution: expression in echinoderms. Eur. J. Biochem. 186: 195 203.

Robin, Y. 1964. Biological distribution of guanidines and phosphagens in marine annelida and related phyla from California. Comp. Biochem. Physiol. 12: 347-367.

Saks, V. A., and M. K. Aliev. 1996. Is there the creatine kinase equilibrium in working heart cells? Biochem. Biophys. Res. Commun 227: $360-367$.

Saks, V. A., E. Vasil'eva, Y. O. Belikova, A. V. Kuznetsov, S. Lyapina, L. Petrova, and N. A. Perov. 1993. Retarded diffusion of ADP in cardiomyocytes; possible role of outer mitochondrial membrane and creatine kinase in cellular regulation of oxidative phosphorylation. Biochim. Biophys. Acta 1144: 134-148.

Saks, V. A., T. Tiivel, L. Kay, V. Novel-Chate, Z. Daneshrad, A. Rossi, E. Fontaine, C. Keriel, X. Leverve, R. Ventura-Clapier, K. Anflous, J.-L. Samuel, and L. Rappaport. 1996. On the regulation of cellular energetics in health and disease. Mol. Cell. Biochem. 160/161: 195-208.

Schneider, A., R. J. Niener, and M. K. Grieshaber. 1989. On the role of arginine kinase in insect flight muscle. Insect Biochem. 19: $471-480$.

Suzuki, T., Y. Kawasaki, T. Furukohri, and W. R. Ellington. 1997. Evolution of phosphagen kinase VI. Isolation, characterization and cDNA-derived amino acid sequence of lombricine kinase from the earthworm Eisenia foetida, and identification of a possible candidate for the guanidine recognition site. Biochim. Biophys. Acta 1343: $152-159$.

Thoai, N. V., N. V. Thien, G. Lacombe, and J. Roche. 1966. Heteroenzymes d'acide adenosine 5'-triphosphoric: L-arginine phosphotransferase. Biochim. Biophys. Acta 122: 547-550.

Thoai, N. V., Y. Robin, and Y. Guillou. 1972. A new phosphagen, 
N'Phosphorylguanidinoethylphospho-O-(a-N,N-dimethyl)serine (phosphothalassemine). Biochem. 11: 3890-3895.

Tombes, R. M., and B. M. Shapiro. 1985. Metabolite channelling: a phosphocreatine shuttle to mediate high energy phosphate transport between sperm mitochondrion and tail. Cell 41: 325-334.

Tombes, R. M., and B. M. Shapiro. 1989. Energy transport and cell polarity: relationship of phosphagen kinase activity to sperm function. J. Exp. Zool. 251: 82-90.

Tombes, R. M., C. J. Brokow, and B. M. Shapiro. 1985. Creatine kinase dependent energy transport in sea urchin spermatozoa. Biophys. J. 52: 75-86.

van Dorsten, F. A., M. Wyss, T. Wallimann, and K. Nicolay. 1997. Activation of sea urchin sperm motility is accompanied by an increase in the creatine kinase flux. Biochem. J. 325: 411-416.

Wallimann, T., M. Wyss, D. Brdiczka, K. Nicolay, and H. M. Eppenberger. 1992. Intracellular compartmentation, structure and function of creatine kinase isoenzymes in tissues with high and fluctuating energy demands: the 'phosphocreatine' circuit. Biochem. J. 281: $21-40$.

Watts, D. C. 1968. The origin and evolution of the phosphagen phosphotransferases. Pp. 279-296 in Homologous Enzymes and Biochemical Evolution, N. V. Thoai and J. Roche, eds. Gordon and Breach, New York.
Watts, D. C. 1971. Evolution of phosphagen kinases. Pp. 150-173 in Biochemical Evolution and the Origin of Life, E. Schoffenials, ed. North-Holland, Amsterdam.

Watts, D. C. 1975. Evolution of phosphagen kinases in the chordate line. Symp. Zool. Soc. Lond. 36: 105-127.

Wothe, D. D., H. Charbonneau, and B. M. Shapiro. 1990. The phosphocreatine shuttle of sea urchin sperm: flagellar creatine kinase resulted from a gene triplication. Proc. Natl. Acad. Sci. USA 87: 5203-5207.

Wu, D., A. Chen, and C. S. Johnson, Jr. 1995. An improved diffusion-ordered spectroscopy experiment incorporating bipolar gradient pulses. J. Magn. Reson. 115A: 260-264.

Wyss, M., J. Smeitnik, R. A. Wevers, and T. Wallimann. 1992. Mitochondrial creatine kinase: a key enzyme of aerobic energy metabolism. Biochim. Biophys. Acta 1102: 119-166.

Wyss, M., D. Maughan, and T. Wallimann. 1995. Re-evaluation of the structure and physiological function of guanidino kinases in the fruit fly (Drosophila), sea urchin (Psammechinus miliaris) and man. Biochem. J. 309: 255-261.

Yoshizaki, K., H. Nishikawa, and H. Watari. 1987. Diffusivities of creatine phosphate and ATP in an aqueous solution studied by pulsed field gradient ${ }^{31}$ P NMR. Jpn. J. Physiol. 37: 923-928. 